\title{
Would endoscopic surgery be the gold standard for stapes surgery in the future? A systematic review and meta-analysis
}

\author{
Lucheng Fang ${ }^{1} \cdot$ Jiayuan $\mathrm{Xu}^{2} \cdot$ Wen $\mathrm{Wang}^{1} \cdot$ Yideng Huang $^{1}$ (])
}

Received: 19 May 2020 / Accepted: 12 June 2020 / Published online: 9 July 2020

(c) The Author(s) 2020

\begin{abstract}
Objective This meta-analysis is aimed to review and analyze all available data of intraoperative and postoperative results of endoscopic and microscopic stapes surgery.

Methods According to the PRISMA statements checklist, this systematic review and meta-analysis were designed. Data were extracted from public databases, such as PubMed, Cochrane, Web of Science, and more. The quality of studies was evaluated using the MINORS scale. Odds ratios (ORs) and 95\% CIs were estimated for binary outcome data, while the mean differences and $95 \%$ CIs were estimated for continuous data. $I^{2}$ and $\chi^{2}$ tests were used to quantify statistical heterogeneity. If more than ten studies were included in each analysis, funnel plot would be performed to analysis publication bias.

Results Twelve studies with 620 patients were included in this meta-analysis. Primary outcomes collected in this metaanalysis included average postoperative auditory gain (APAG), postoperative air-bone gap (ABG), the rate of chorda tympani handling and bone curettage, which all showed a statistically significant difference in favor of endoscopy. While only secondary outcomes about postoperative pain and dysgeusia demonstrated a significantly reduced incidence. Furthermore, there was not any statistically significant difference on postoperative dizziness and average operative time between endoscopy and microscopy.

Conclusion Although there is a need for high-quality pooled data in the future, a consistently superior effect of the endoscopic group was still shown in terms of total effectiveness, when compared to the microscopic group. We have reasons to support the application of endoscopy in stapes surgery. The future of ESS, we believe, is blazing bright.
\end{abstract}

Keywords Endoscopic $\cdot$ Microscopic $\cdot$ Otosclerosis $\cdot$ Congenital stapedial fixation $\cdot$ Stapes surgery

\section{Introduction}

Traditionally, microscopic surgery is the best choice to perform stapes surgery (SS), since Shea first described modern stapedectomy with a teflon implant in 1956 [1-3]. Although microscopic stapes surgery (MSS) nowadays is quite successful, some limitations still certainly exist. Primarily, the visualization of the tympanic membrane and middle-ear structures is limited, especially for patients who have narrow segment of the external auditory canal, because of the perennial problem of the tunnel vision which often contributes

Yideng Huang

huang_yideng@126.com

1 First Affiliated Hospital of Wenzhou Medical University, Wenzhou City, Zhejiang Province, China

2 Wenzhou Medical University, Wenzhou City, Zhejiang Province, China to the requirement of an external incision $[1,4,5]$. Consequently, endoscopic stapes surgery (ESS) is gaining growing attention all over the world. The main reason for this booming interest is the patients' wish for minimal invasive surgery to avoid a painful incision. What's more, advantages of ESS, such as providing better surgical field exposure with panoramic vision to hidden recesses, as well as complex structures, and reduced bone curettage, also satisfy the demand of surgeons. Additionally, the endoscope could lightly pass a narrow isthmus in the transcanal approach, resulting in the enhanced visualization of the ossicular chain and oval window clear [6-9]. Nevertheless, the endoscope is not perfect. The lack of depth perception because of two-dimensional vision, the operation of one hand, and the relatively long learning curve all make many otologists unwilling to switch to endoscopic technology [9-11]. Despite the awkward difficulty of endoscopic one-hand surgery, almost all of the 
surgical steps, theoretically speaking, involved in the stapes surgery procedures were practicable by endoscopy.

The SS is mainly applied to otosclerosis and congenital stape footplate fixation (CSFF), frequently presenting with an intact tympanic membrane and acquired conductive hearing loss, and stapedotomy has been widely regarded as the most efficient surgical modality for treatment $[1,3$, 12]. Some previous clinical studies have indicated that an endoscopic approach, when compared with a traditional microscopic approach, has almost, even completely, similar hearing outcomes, while both rates of chorda tympani nerve(CTN) transection, as a matter of fact, and scutum removal are decreased likewise [2, 3, 7, 8, 13]. However, recognizing that individual studies probably cannot provide sufficient evidence on their own to affect clinical practice, we sought to objectively assess the difference between ESS and MSS. Therefore, this new systematic review and metaanalysis was reported to search and analysis all clinical data from available controlled studies, so that the similarities and differences between ESS and MSS could be compared in various aspects.

\section{Methods}

\section{Study design and inclusion criteria}

According to the PRISMA statements checklist [14], this systematic review and meta-analysis were performed.

Meticulously, we defined inclusion criteria before initiating the data search to ensure that all eligible studies are correctly identified. The study selection procedures were performed independently by two reviewers (X.J.Y. and W.W.) and disagreements were also resolved by consensus. The PICO (population, intervention, comparison, and outcome) framework applied as the selection criteria were as follows: (1) the population included patients with otosclerosis or congenital stapedial fixation. (2) Only controlled studies comparing ESS and MSS were analyzed. Case reports, case series, commentaries, conference abstracts, and review articles were not considered. (3) Postoperative outcomes, such as the average postoperative air-bone gap (ABG), the manipulation of CTN, postoperative dizziness and so on, were documented sufficiently [15].

\section{Literature search strategy}

A literature search was performed in the PubMed, Cochrane, Web of Science, ClinicalTrials and EMBASE from inception up to January 1, 2020. We applied the following freetext terms to searching in the PubMed database: (otoscleros* OR otospongios* OR "congenital stapedial fixation" OR “congenital stapes fixation") AND (stapedotom* OR stapedectom* OR "stapes surgery") AND (endoscop* OR microscop*). In addition, we modified this search strategy to apply to search rules of other databases. Besides, we independent reviewers, to identify additional suitable studies, cross-checked both the reference lists of the available studies and relevant review articles.

\section{Data extraction}

Two reviewers (X.J.Y. and W.W.) screened both the title and abstract, independently and blindly, of studies collected. For enhancing sensitivity of analysis, only when both reviewers excluded the record at the title and abstract screening level, could this record be definitely removed. A full-text analysis of the selected articles, subsequently, was performed by both reviewers. It was, if necessary, a third reviewer (F.L.C.) that resolved any divergence between the two reviewers in the selection and evaluation procedures. The following data from each selected study were extracted: characteristics (lead author, year of publication, country, study design, the amount of patients, study population, mean age, and mean length of follow-up) and outcomes data. Two independent reviewers assessed risk for bias according to the PRISMA recommendations and the Cochrane tool for assessing risk for bias $[14,16]$. Subsequently, we made the qualitative and quantitative analyses of all data from the involved studies.

\section{Statistical analysis}

We assessed the effect of ESS and MSS on primary and secondary outcomes, and divided the above data into continuous data and binary data. For continuous data, the mean differences [standard mean differences (SMD) or weighted mean difference (WMD)] and 95\% CIs were estimated. For binary outcome data, odds ratios (ORs) and 95\% CIs were estimated using the Mantel-Haenszel method [16]. What's more, the Cochran $Q$ test was used to evaluate heterogeneity [17]. Also, $I^{2}$ testing was applied to evaluate the magnitude of the heterogeneity between studies. If $I^{2} \leq 50 \%$, the fixed effects model was used for analysis, or the random effects model would be chosen [16, 18]. We used Stata (version 12.0 SE) for all statistical analysis.

\section{Result}

\section{Study selection and characteristics}

Totally, 1314 articles were identified, of which 178 were excluded because of duplication. After evaluating the title and abstract, 924 articles were removed. The full-text of the remaining 212 articles was reviewed, and we also contacted some authors to ask for unpublished data. For the 
qualitative and quantitative analysis, only 12 studies eventually remained. It is in Fig. 1 that thoroughly shows the PRISMA diagram of the literature search and selection procedures. All selected studies, including 673 patients undergoing ESS or MSS, were written in English and published from 2014 to 2019. Two studies were randomized controlled trials (RCTs), and the others were non-randomized cohort studies (nRCSs). Patients who underwent ESS belonged to the exposure group, and the control group included all patients who underwent MSS. The detailed information of the 12 trials was partially exhibited in Table 1.

\section{Quality assessment}

The MINORS Scale, developed for randomized surgical trials and observational studies in surgery by a group of surgeons, was applied to evaluating the methodological quality of included studies [19]. 12 items were included and the items are scored 0 (not reported), 1 (reported but inadequate) or 2 (reported and adequate). According to the aggregate scores of each study, we allocated different score ranges a "high" (0-8), "middle" (9-16) and "low" (17-24) risk of bias, because of the lack of explicit definition in the
MINORS Scale. The exact scoring of individual studies was presented in Table 2. Ultimately, four studies had low risk of bias while eight studies had middle risk of bias. The main reasons of risk of bias, as the table exhibited, were the lack of consecutive patients, non-blind trails and the absence of prospective calculation of the study size. Fortunately, no studies of high risk of bias were included.

\section{Primary outcomes}

Eight studies [2, 3, 6-8, 20-22] recorded the outcome of average postoperative auditory gain (APAG), but only five articles $[2,6,8,20,22]$ have reported both the value of mean and standard deviation. The standard mean difference (SMD) of APAG was 0.44 [95\% CI $0.21-0.67 ; P=0.441$; heterogeneity, $I^{2}=0 \%$ ] (Fig. 2a). Because of $I^{2} \leq 50 \%$, we chose fixed effects models for analysis. Obviously, there was a statistically significant difference in favor of ESS. Seven studies $[3,6,12,13,21,23,24]$ recorded the outcome of postoperative air-bone gap (ABG) of $10 \mathrm{~dB}$ or less. The OR was 1.99 [95\% CI 1.26-3.13; $P=0.6 .28$; heterogeneity, $I^{2}=0 \%$ ] (Fig. 2b), and fixed effects models were applied to analysis. Significant difference also existed statistically in
Fig. 1 The flowchart shows the literature search and study selection process according to the PRISMA guidelines
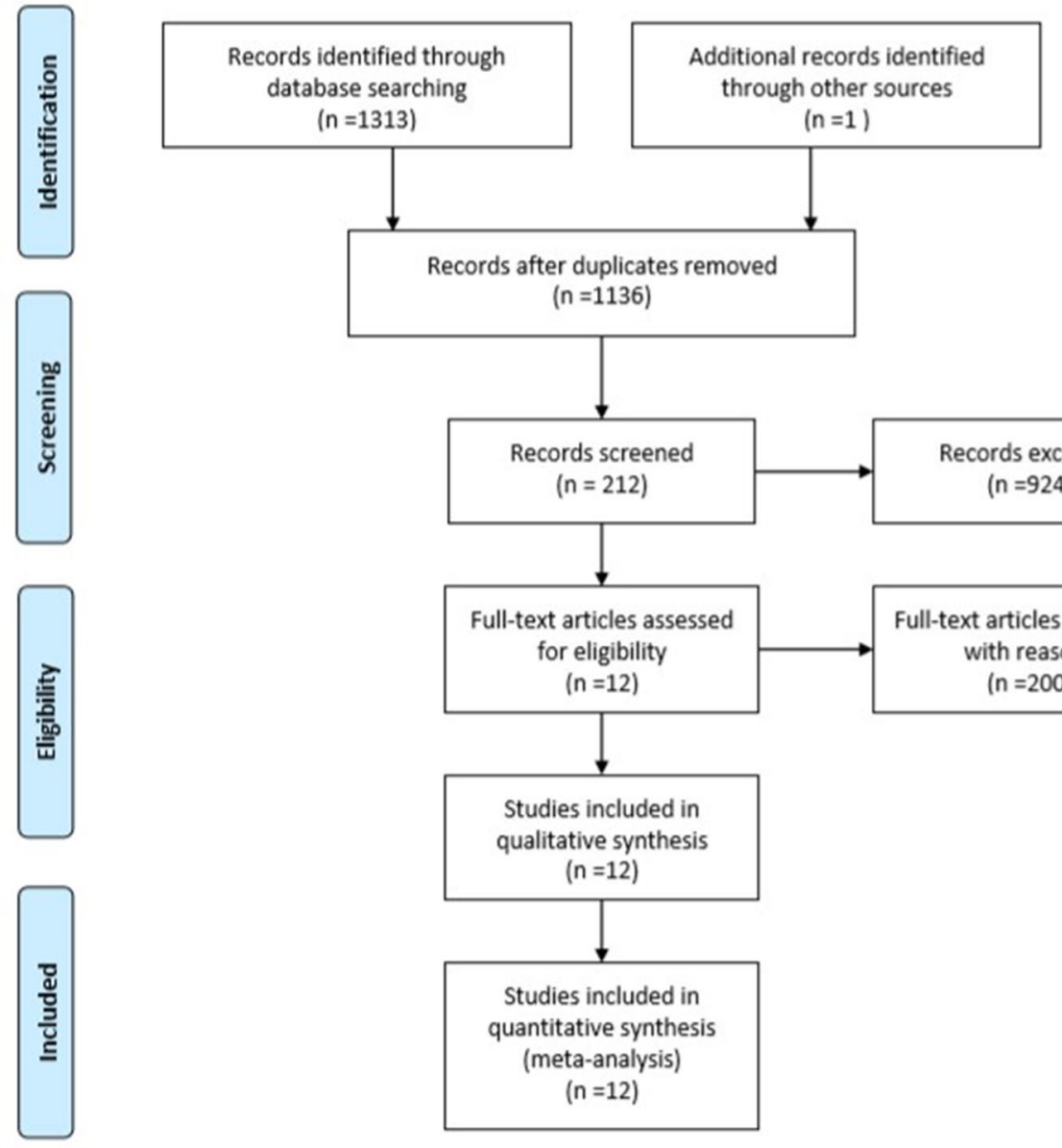
( $n=1136$ )
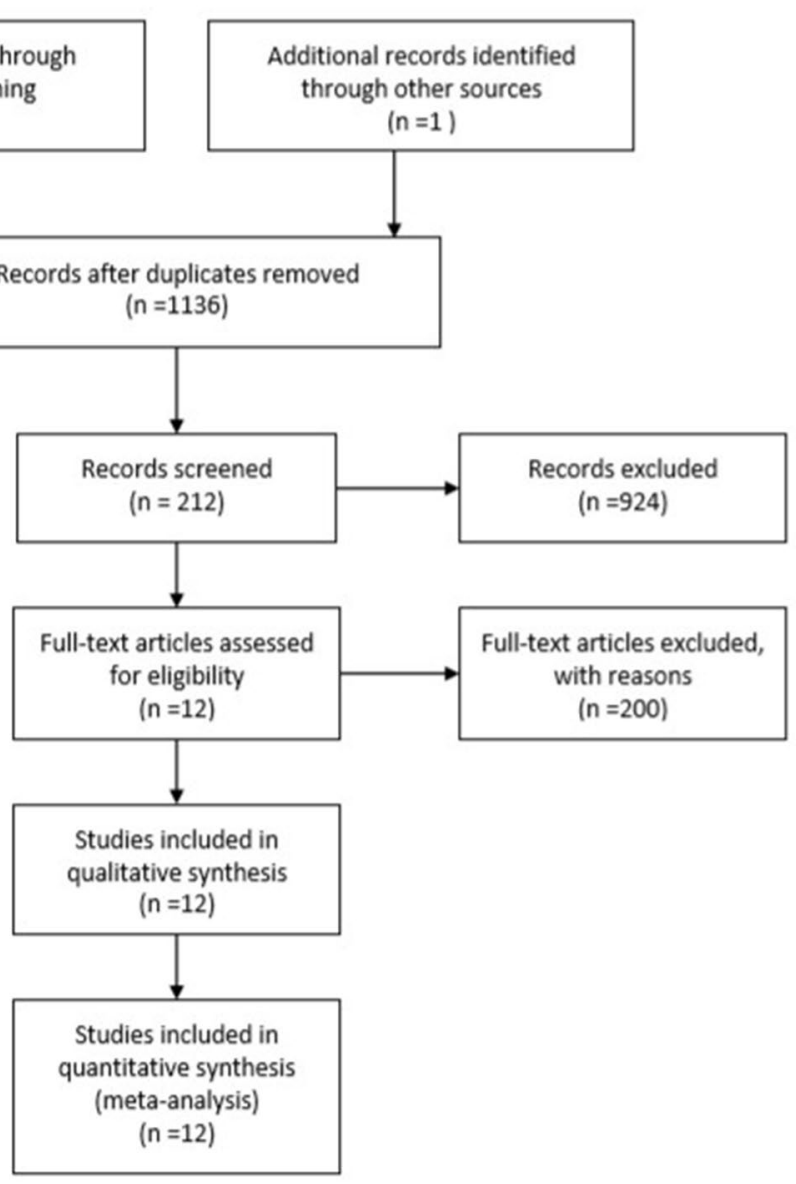
Table 1 Study characteristics

\begin{tabular}{|c|c|c|c|c|c|c|c|c|c|c|c|}
\hline \multirow[t]{2}{*}{ Lead author (year) } & \multirow[t]{2}{*}{ Country } & \multirow[t]{2}{*}{ Study design } & \multicolumn{3}{|c|}{ Patients (no.) } & \multirow[t]{2}{*}{ Study population } & \multicolumn{2}{|c|}{$\begin{array}{l}\text { Mean age } \\
\text { (years) }\end{array}$} & \multicolumn{2}{|c|}{$\begin{array}{l}\text { Mean } \\
\text { follow-up } \\
\text { (month) }\end{array}$} & \multirow[t]{2}{*}{ Outcomes } \\
\hline & & & Total & ESS* & MSS* & & EDG & MCG & EDG & $\mathrm{MCG}$ & \\
\hline Kojima et al. (2014) [12] & Japan & $\mathrm{nRCS}$ & 56 & 15 & 41 & $\begin{array}{c}\text { Otosclerosis and } \\
\text { congenital sta- } \\
\text { pedial fixation }\end{array}$ & 40.1 & 49.47 & 8.6 & 8.6 & $\mathrm{a}, \mathrm{e}$ \\
\hline Daneshi et al. (2015) & Iran & $\mathrm{nRCS}$ & 34 & 19 & 15 & Otosclerosis & 36.8 & 36.8 & 7.42 & 7.42 & $\mathrm{a}, \mathrm{b}, \mathrm{d}, \mathrm{e}$ \\
\hline Iannella et al. (2016) [24] & Italy & $\mathrm{nRCS}$ & 40 & 20 & 20 & Otosclerosis & 44.3 & 45.3 & 10.3 & 9 & $\mathrm{a}, \mathrm{b}, \mathrm{c}, \mathrm{d}, \mathrm{e}$ \\
\hline Sproat et al. (2017) [23] & Turkey & $\mathrm{nRCS}$ & 81 & 34 & 47 & Otosclerosis & 47 & 51 & 5 & 10 & $\mathrm{a}, \mathrm{c}$ \\
\hline Surmelioglu et al. (2017) [22] & UK & $\mathrm{nRCS}$ & 46 & 22 & 24 & Otosclerosis & 39.2 & 44.3 & 15.8 & 21.5 & $\mathrm{a}, \mathrm{b}, \mathrm{c}, \mathrm{d}, \mathrm{e}$ \\
\hline Moneir et al. (2018) [21] & USA & $\mathrm{nRCS}$ & 42 & 22 & 24 & Otosclerosis & 33.6 & 33 & 4.5 & 5.5 & $\mathrm{a}, \mathrm{d}, \mathrm{e}$ \\
\hline Ardic et al. (2018) [6] & India & $\mathrm{nRCS}$ & 94 & 37 & 57 & Otosclerosis & 42.2 & 43.8 & 12 & 12 & $\mathrm{a}, \mathrm{e}$ \\
\hline Bhardwaj et al. (2018) [8] & Egypt & RCT & 40 & 20 & 20 & Otosclerosis & 33.1 & 32.9 & 12 & 12 & $\mathrm{a}, \mathrm{b}, \mathrm{c}, \mathrm{d}, \mathrm{e}$ \\
\hline Kuo et al. (2018) [7] & China Taipei & $\mathrm{nRCS}$ & 30 & 17 & 13 & Otosclerosis & 49.6 & 54.2 & $\mathrm{~N}$ & $\mathrm{~N}$ & $\mathrm{a}, \mathrm{b}$ \\
\hline Gulsen et al. (2019) [2] & Turkey & $\mathrm{nRCS}$ & 72 & 38 & 34 & Otosclerosis & 32.6 & 35.6 & 13.2 & 12.1 & $\mathrm{a}, \mathrm{b}, \mathrm{c}, \mathrm{d}, \mathrm{e}$ \\
\hline Tolisano et al. (2019) [3] & USA & $\mathrm{nRCS}$ & 74 & 22 & 52 & Otosclerosis & 10.5 & 10.5 & 7.1 & 27 & $\mathrm{a}, \mathrm{b}, \mathrm{c}, \mathrm{d}, \mathrm{e}$ \\
\hline Das et al. (2019) [20] & India & $\mathrm{RCT}$ & 64 & 32 & 32 & $\begin{array}{c}\text { Otosclerosis and } \\
\text { congenital sta- } \\
\text { pedial fixation }\end{array}$ & 40 & 38.5 & 1 & 1 & $\mathrm{a}, \mathrm{d}, \mathrm{e}$ \\
\hline
\end{tabular}

*Items of evaluation: ESS endoscopic stapes surgery, MSS microscopic stapes surgery

${ }^{\text {a }}$ Postoperative hearing

${ }^{\mathrm{b}}$ Scutum drilling

${ }^{\mathrm{c}}$ Chorda tympani handling

${ }^{\mathrm{d} O p e r a t i o n ~ t i m e ~}$

${ }^{\mathrm{e}}$ Postoperative complications

Table 2 Risk of bias analysis

\begin{tabular}{llllllllllllll}
\hline Study & $1 *$ & 2 & 3 & 4 & 5 & 6 & 7 & 8 & 9 & 10 & 11 & 12 & Total \\
\hline Kojima et al. (2014) [12] & 2 & 0 & 0 & 1 & 0 & 2 & 1 & 0 & 2 & 0 & 1 & 1 & 10 \\
Daneshi et al. (2015) & 2 & 0 & 0 & 1 & 0 & 2 & 1 & 0 & 2 & 0 & 1 & 1 & 10 \\
Iannella et al. (2016) [24] & 2 & 0 & 0 & 1 & 0 & 2 & 1 & 0 & 2 & 2 & 1 & 1 & 12 \\
Sproat et al. (2017) [23] & 2 & 0 & 2 & 1 & 0 & 2 & 1 & 0 & 2 & 0 & 1 & 1 & 12 \\
Surmelioglu et al. (2017) [22] & 2 & 0 & 0 & 1 & 0 & 2 & 1 & 0 & 2 & 0 & 1 & 2 & 11 \\
Moneir et al. (2018) [21] & 2 & 0 & 2 & 1 & 0 & 2 & 1 & 0 & 2 & 0 & 1 & 2 & 15 \\
Ardic et al. (2018) [6] & 2 & 0 & 0 & 1 & 0 & 2 & 1 & 0 & 0 & 2 & 1 & 1 & 10 \\
Bhardwaj et al. (2018) [8] & 2 & 2 & 2 & 2 & 1 & 2 & 2 & 0 & 2 & 2 & 1 & 1 & 19 \\
Kuo et al. (2018) [7] & 2 & 1 & 1 & 2 & 0 & 0 & 1 & 0 & 2 & 0 & 1 & 2 & 12 \\
Gulsen et al. (2019) [2] & 2 & 2 & 2 & 2 & 1 & 2 & 1 & 0 & 2 & 1 & 1 & 2 & 18 \\
Tolisano et al. (2019) [3] & 2 & 2 & 1 & 2 & 0 & 2 & 1 & 0 & 2 & 2 & 1 & 2 & 17 \\
Das et al. (2019) [20] & 2 & 2 & 2 & 2 & 1 & 1 & 2 & 0 & 2 & 2 & 1 & 2 & 19 \\
\hline
\end{tabular}

*Items of evaluation: 1 a clearly stated aim, 2 inclusion of consecutive patients, 3 prospective collection of data, 4 endpoints appropriate to the aim of the study, 5 unbiased assessment of the study endpoint, 6 follow-up period appropriate to the aim of the study, 7 loss to follow-up less than $5 \%, 8$ prospective calculation of the study size, 9 an adequate control group, 10 contemporary groups, 11 baseline equivalence of groups, 12 adequate statistical analyses

favor of ESS. Besides, when excluding the outcome of Tolisano's study [3] resulting from patients who were all children with narrow meatus, the statistically significant difference is obvious. The OR of pooling data, containing five studies
$[2,8,22-24]$, of chorda tympani handling was 0.22 [95\% CI 0.11-0.42; $P=0.944$; heterogeneity, $I^{2}=0 \%$ ] (Fig. 2c). The $I^{2}$ statistical analysis suggested mild heterogeneity, and the rate of chorda tympani handling is significantly higher 

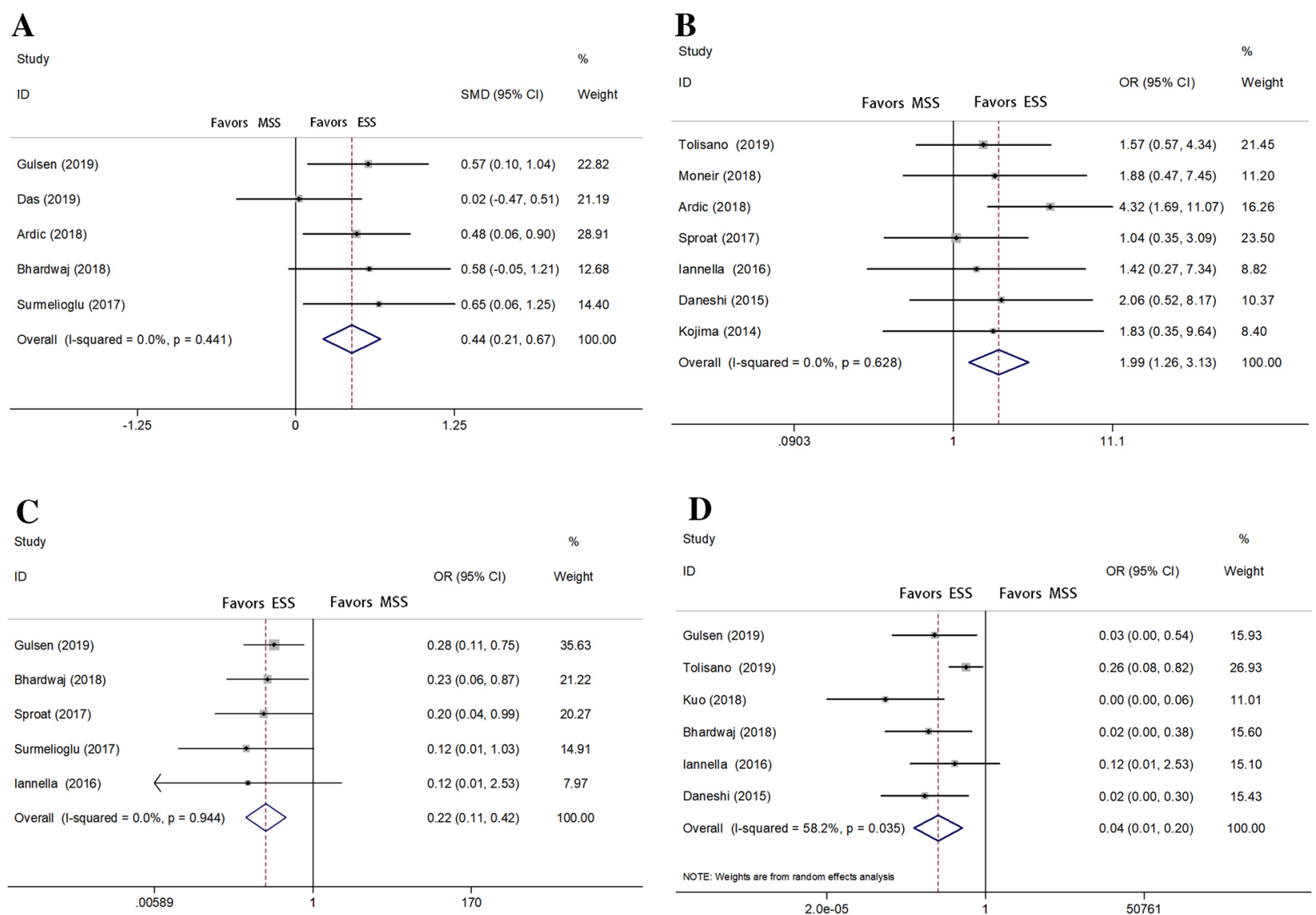

Fig. 2 Forest plots with respect to primary outcomes (a average postoperative auditory gain b postoperative ABG of $10 \mathrm{~dB}$ or less $\mathbf{c}$ chorda tympani handling $\mathbf{d}$ bone curettage)

with MSS. Similarly, the rate of bone curettage was reduced by $96 \%$ with endoscope. The OR, favoring ESS apparently, was 0.04 [95\% CI 0.01-0.20; $P=0.035$; heterogeneity, $I^{2}=58.2 \%$ ] (Fig. 2d), and a random effects model was employed because of $I^{2} \geq 50 \%[2,3,7,8,13,24]$.

\section{Secondary outcomes}

The number of postoperative dizziness was recorded in seven studies [2, 3, 8, 13, 21, 22, 24], and Iannella's study [24] was removed automatically by software because of the same outcomes in two groups. The OR was 0.69 [95\% CI $0.34-1.41 ; P=0.970$; heterogeneity, $I^{2}=0 \%$ ] in fixed effects models. There was no statistically significant difference between ESS and MSS (Fig. 3a). Besides, the outcomes of postoperative pain $[2,8,20,22,24]$ and postoperative dysgeusia $[2,12,20,22,24]$ were respectively recorded in five studies. The OR of pain was 0.30 [95\% CI 0.16-0.59; $P=0.698$; heterogeneity, $I^{2}=0 \%$ ] (Fig. 3b) and the OR of dysgeusia was 0.21 [95\% CI 0.11-0.41; $P=0.205$; heterogeneity, $I^{2}=32.5 \%$ ] (Fig. 3c). Both of them favored ESS with the statistically significant difference. Nine studies compared the average operative time $[2,3,7,8,13,20-22,24]$, but only four studies were included resulting from the paucity of the standard deviation [2, 7, 20,22]. No significant difference was showed between the two operative approaches [WMD $=-7.364 ; 95 \% \mathrm{CI}-26.50-11.77 ; P=0.451$; heterogeneity, $I^{2}=98.9 \%$ ] (Fig. 3d). According to the value of $I^{2}$, the heterogeneity is considerable. We did not perform any funnel plot, since less than ten studies were included in each analysis.

\section{Discussion}

Undoubtedly, endoscopes, an promising technique, is superior to microscopic surgery for considerable benefits, such as improved surgical field and panoramic view of the complex middle ear structure, leading to fewer bone removal and, to some extent, less injury to CTN according to previous experience [5]. In this meta-analysis, we pooled the largest amount of record to date with direct comprehensive comparison of ESS and MSS since 2014 to 2019. 


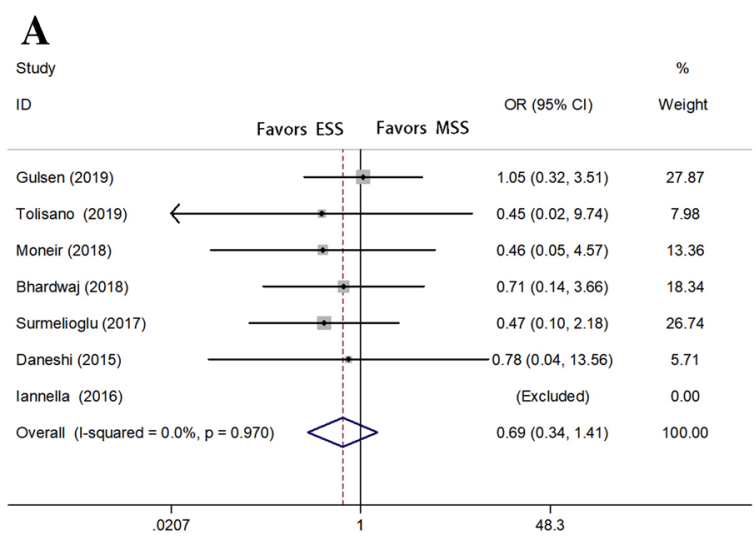

\section{B}

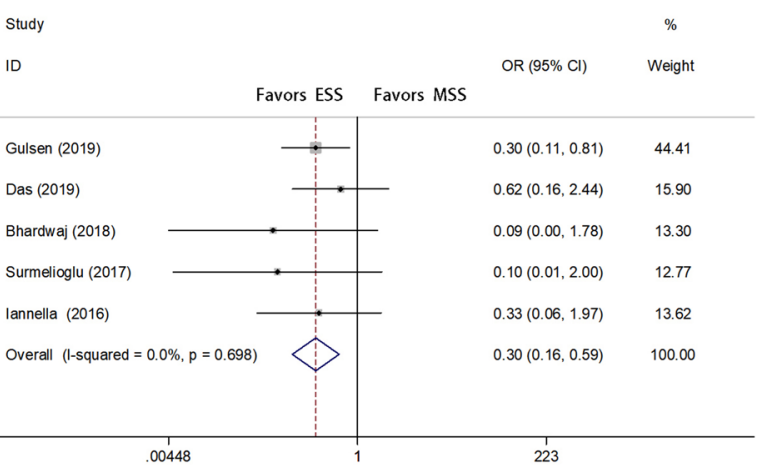

C

D
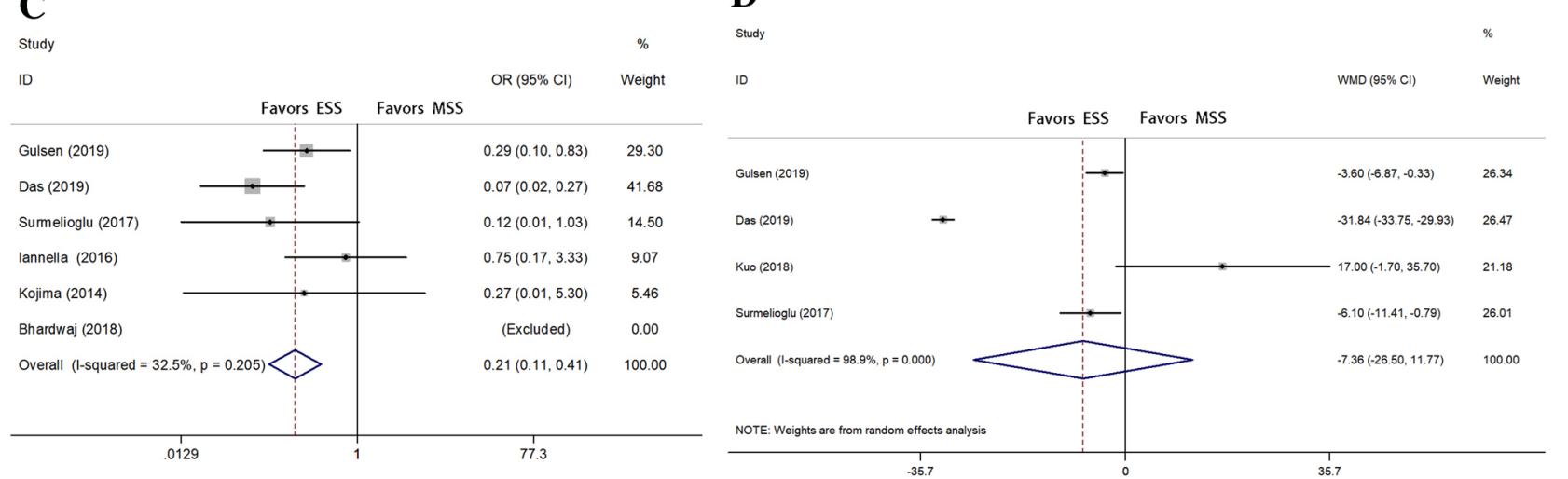

Fig. 3 Forest plots with respect to secondary outcomes (a postoperative dizziness b postoperative pain $\mathbf{c}$ dysgeusia $\mathbf{d}$ average operative time)

The situation of postoperative hearing is the most valuable item to measure whether the surgery is successful. To the best of our knowledge, the item, APAG, was firstly evaluated in our meta-analysis. As can be observed, pooled data based on five studies showed a significantly less value of postoperative $A B G$ in ESS. On the other hand, closure of the ABG to $<10 \mathrm{~dB}$ is a widely reported outcome measure for success in stapes surgery [23]. Unlike previous two meta-analyses [4, 5], it is a statistical difference favoring the endoscope that exists in our study. The outcome of postoperative hearing obviously indicated that a superiority of ESS over MSS is indeed present.

Extent of chorda tympani manipulation, to be sure, is also an imperative, even incredibly difficult, point in stapes surgery [25-27]. As expected, our study has reached statistical significance in favor of the endoscope. Thanks to angled endoscopes, wider panoramic view of the surgical field, when accessed from inferior to superior direction from the hypotympanum, was provided in ESS $[9,11]$. But in the microscopic group, it was ineluctable to manipulate CTN because of limited visualization. However, in light of all patients who were children with narrow external canal, we removed the results of Tolisano et al. [3], or no statistical difference would exist in our analysis.
As far as we know, alteration in taste sensation is directly associated with tympani manipulation during surgery [25, 28]. According to reports before, many patients who underwent MSS suffered from postoperative taste disturbance, which can be present in 20-60\% of patients after this surgery $[22,28]$. In contrast, some authors reported that endoscopic surgery, for avoiding handling the chorda tympani, may result in a reduced incidence of postoperative dysgeusia $[24,26]$. In our analysis including six studies, we find statistical difference supporting the above conclusion similar to Nikolaos's et al. outcome [4] but different from Koukkoullis's et al. analysis [5].

Adequate exposure is critical both in ESS and in MSS. The visibility of the footplate area is crucial to ensure accurate insertion of the piston and minimal invasion to the fragile inner ear structures [8, 9, 29]. Yet, in MSS, the postero-superior bony canal wall often encumbers the view of the surgical field so that bony canal wall has to be removed for better visualization. Conversely, the incidence of extensive bone curettage or drilling was minimal in ESS, and wider panoramic view is obtained with fewer sacrifice of any soft tissue lying ahead [9]. What's more, it was fewer patients, after endoscopic stapedotomy, that complained of postoperative pain $[11,20]$. Indeed, our 
meta-analysis, in accordance with these recent findings, showed a statistically significant difference. On the contrary, a prior meta-analysis based on four retrospective studies which was published in 2018 showed, in the main, no differences in postoperative pain between the endoscopic and microscopic approaches, but we should not neglect that the most recent studies were not included [4]. Therefore, we have reasons to believe that our latest conclusion is more credible.

Postoperative dizziness was also a substantial complaint affecting the patients' quality of life after surgery. Patients performed with ESS would suffer, theoretically speaking, less with postoperative dizziness because of, it is thought, reduced operation of the stapes footplate due to better visualization. Nevertheless, no significant difference was found in our analysis. In light of the above outcome, we postulate that the endoscope temperature probably could affect the inner ear leading to unknown influence in spite of the minimally invasive approach [30]. What's more, postoperative dizziness, in our opinion, is mainly related to the trauma severity of fenestration and the length of the prosthesis rather than the type of surgical approach. Obviously, further studies are needed to ascertain our hypothesis.

Theoretically, the endoscopic operative field was clear and otologic surgeons could easily identify anatomy of the middle ear leading to sacrifice fewer bony structures or even not, which could, to some extent, shorten the operative time. Conversely, the operative time, in some studies $[3,7]$, is longer for endoscopic than microscopic surgeries whether surgeons are novice or experienced, implying this is not simply due to the "learning curve". Admittedly, surgeons also spent additional time on continually wiping the fogged or blood-stained endoscope or on stopping bleeding with onehanded surgery. Therefore, there is a need for high-quality pooled data in the future to fully compare ESS with MSS on operative time.

Despite the aforementioned positive conclusions of endoscopic stapes surgery, the limitations of endoscopy, such as only one hand operation, the two-dimensional vision leading to lack of depth perception and potentially long learning curve, must be weighed in the determination of introducing endoscopic technology in stapes surgery $[9,11,30]$. To get rid of above limitations, the use of a robotic system to hold endoscope, and the application of $3 \mathrm{D}$ endoscopes to provide stereoscopic vision was recommended $[10,31]$. But those equipment are too immature to be applied. As a matter of fact, with the experience of surgery increasing gradually, the otologic doctors are accustomed to working with the two-dimensional vision, so that the surgical manipulation will be no longer limited by the lack of stereoscopic vision $[8,24]$. On the other hand, the patient's postoperative health and, of course, quality of life, like taste disorder, pain and the requirement of minimally invasive surgery, are supposed to be considered and ESS seems to be superior to MSS in those areas.

There are, to be sure, still some limitations in this study. First, a limitation of this analysis is that only two RCTs were included while others were nRCSs, which is almost usually regarded as a relatively low level of evidence. Therefore, it is, in a way, nearly impossible to eliminated potential bias completely. For example, surgical procedures, among all of involved studies, were not standardized, and surgery in some studies was even performed by different surgeons. This bias may result in an underestimation of the efficacy of the experimental procedure. What's more, children who were patients with congenital stapedial fixation were also included in our study. This study population, undoubtedly, would result in a falsely decreased efficacy, since these cases, generally speaking, are related to poor postoperative hearing outcomes and a higher incidence of complications. Additionally, further bias is the size of prosthesis. According to a previous meta-analysis, Laske et al. found it more likely for $0.6 \mathrm{~mm}$ pistons than for $0.4 \mathrm{~mm}$ pistons to gain an air-bone gap closure of less than $10 \mathrm{~dB}$ [29]. The discrepancy in the size of prosthesis might, to some extent, affect the outcomes of postoperative hearing. Besides, APAG can be influenced by the preoperative air-bone gap leading to potential bias. Additionally, endoscopic groups had relatively shorter follow-up than microscopic groups as this new technique was just adopted for a while. Despite these limitations, the sensitivity analyses and the low heterogeneity observed, as seen here, supported the robustness and consistency of our results. The deduction, to put it differently, of our study is valuable.

\section{Conclusion}

In spite of the fact that, admittedly, some of the including studies had small sample sizes and relatively poor methodological quality, a consistently superior effect of the ESS group, in terms of total effectiveness, was still showed in the analysis of the pooled data, when compared to the MSS group. Although further studies are needed, our findings clearly support the use of endoscopy in stapes surgery. The future of ESS, we believe, is blazing bright.

\section{Compliance with ethical standards}

Conflict of interest The authors have no conflict of interest.

Ethical approval Not required.

Informed consent Not required. 
Open Access This article is licensed under a Creative Commons Attribution 4.0 International License, which permits use, sharing, adaptation, distribution and reproduction in any medium or format, as long as you give appropriate credit to the original author(s) and the source, provide a link to the Creative Commons licence, and indicate if changes were made. The images or other third party material in this article are included in the article's Creative Commons licence, unless indicated otherwise in a credit line to the material. If material is not included in the article's Creative Commons licence and your intended use is not permitted by statutory regulation or exceeds the permitted use, you will need to obtain permission directly from the copyright holder. To view a copy of this licence, visit http://creativecommons.org/licenses/by/4.0/.

\section{References}

1. Nazarian R, McElveen JT Jr, Eshraghi AA (2018) History of otosclerosis and stapes surgery. Otolaryngol Clin North Am 51:275-290

2. Gulsen S, Karatas E (2019) Comparison of surgical and audiological outcomes of endoscopic and microscopic approach in stapes surgery. Pak J Med Sci 35:1387-1391

3. Tolisano AM, Fontenot MR, Nassiri AM et al (2019) Pediatric stapes surgery: hearing and surgical outcomes in endoscopic vs microscopic approaches. Otolaryngol Head Neck Surg $161: 150-156$

4. Nikolaos T, Aikaterini T, Dimitrios D et al (2018) Does endoscopic stapedotomy increase hearing restoration rates comparing to microscopic? A systematic review and meta-analysis. Eur Arch Otorhinolaryngol 275:2905-2913

5. Koukkoullis A, Toth I, Gede N, Szakacs Z (2019) Endoscopic versus microscopic stapes surgery outcomes: a meta-analysis and systematic review. Laryngoscope

6. Ardiç FN, Aykal K, Tümkaya F et al (2018) Improvement of hearing results by bone cement fixation in endoscopic stapedotomy. $\mathrm{J}$ Laryngol Otol 132:486-488

7. Kuo CW, Wu HM (2018) Fully endoscopic laser stapedotomy: is it comparable with microscopic surgery? Acta Otolaryngol 138:871-876

8. Bhardwaj A, Anant A, Bharadwaj N et al (2018) Stapedotomy using a $4 \mathrm{~mm}$ endoscope: any advantage over a microscope? J Laryngol Otol 132:807-811

9. Isaacson B, Hunter JB, Rivas A (2018) Endoscopic stapes surgery. Otolaryngol Clin North Am 51:415-428

10. Bernardeschi D, Lahlou G, De Seta D et al (2018) 3D endoscopic ear surgery: a clinical pilot study. Eur Arch Otorhinolaryngol 275:379-384

11. Marchioni D, Rubini A, Gazzini L et al (2018) Complications in endoscopic ear surgery. Otol Neurotol 39:1012-1017

12. Kojima H, Komori M, Chikazawa S (2014) Comparison between endoscopic and microscopic stapes surgery. Laryngoscope 124:266-271

13. Daneshi A, Jahandideh H (2016) Totally endoscopic stapes surgery without packing: novel technique bringing most comfort to the patients. Eur Arch Otorhinolaryngol 273:631-634
14. Liberati A, Altman DG, Tetzlaff J et al (2009) The PRISMA statement for reporting systematic reviews and meta-analyses of studies that evaluate healthcare interventions: explanation and elaboration. BMJ (Clinical research ed) 339:b2700

15. Lailach S, Schenke T, Baumann I et al (2018) Living with otosclerosis: disease-specific health-related quality-of-life measurement in patients undergoing stapes surgery. Eur Arch Otorhinolaryngol 275:71-79

16. Higgins JPT, Green S (eds) (2011) Cochrane handbook for systematic reviews of interventions, Version 5.1.0. The Cochrane Collaboration, London

17. Higgins JP, Thompson SG, Deeks JJ, Altman DG (2003) Measuring inconsistency in meta-analyses. BMJ (Clinical research ed) 327:557-560

18. Higgins JP, Thompson SG (2002) Quantifying heterogeneity in a meta-analysis. Stat Med 21:1539-1558

19. Slim K, Nini E, Forestier D et al (2003) Methodological index for non-randomized studies (minors): development and validation of a new instrument. ANZ J Surg 73:712-716

20. Das A, Mitra S, Ghosh D, Sengupta A (2019) Endoscopic stapedotomy: overcoming limitations of operating microscope. Ear Nose Throat J 145561319862216

21. Moneir W, Abd El-Fattah AM, Mahmoud E, Elshaer M (2018) Endoscopic stapedotomy: merits and demerits. J Otol 13:97-100

22. Surmelioglu O, Ozdemir S, Tarkan O et al (2017) Endoscopic versus microscopic stapes surgery. Auris Nasus Larynx 44:253-257

23. Sproat R, Yiannakis C, Iyer A (2017) Endoscopic stapes surgery: a comparison with microscopic surgery. Otol Neurotol 38:662-666

24. Iannella G, Magliulo G (2016) Endoscopic versus microscopic approach in stapes surgery: are operative times and learning curve important for making the choice? Otol Neurotol 37:1350-1357

25. Berling Holm K, Knutsson J, Strömbäck K et al (2017) Taste disturbance after stapes surgery: an evaluation of frequency, severity, duration, and quality-of-life. Acta Otolaryngol 137:39-43

26. Ciofalo A, Zambetti G, Romeo M et al (2015) Taste and olfaction in middle ear surgery. Ann Otol Rhinol Laryngol 124:312-316

27. Ramaswamy AT, Lustig LR (2018) Revision surgery for otosclerosis. Otolaryngol Clin North Am 51:463-474

28. Maeda E, Katsura H, Nin T et al (2018) Change of somatosensory function of the tongue caused by chorda tympani nerve disorder after stapes surgery. Laryngoscope 128:701-706

29. Laske RD, Röösli C, Chatzimichalis MV et al (2011) The influence of prosthesis diameter in stapes surgery: a meta-analysis and systematic review of the literature. Otol Neurotol 32:520-528

30. Mitchell S, Coulson C (2017) Endoscopic ear surgery: a hot topic? J Laryngol Otol 131:117-122

31. Nguyen Y, Bernardeschi D, Sterkers O (2018) Potential of robotbased surgery for otosclerosis surgery. Otolaryngol Clin North Am 51:475-485

Publisher's Note Springer Nature remains neutral with regard to jurisdictional claims in published maps and institutional affiliations. 